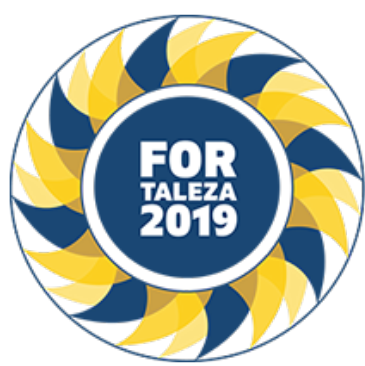

\title{
LIMB CLAUDICATION SYMPTOMS AND VESSEL IMAGING IN ADULT TAKAYASU ARTERITIS: IS THERE A
} RELIABLE CORRELATION?

Carlo Scognamiglio Renner Araujo (Universidade de São Paulo, São Paulo, SP, Brasil), Alexandre Moura dos Santos (Universidade de São Paulo, São Paulo, SP, Brasil), Pablo Arturo Olivo Pallo (Universidade de São Paulo, São Paulo, SP, Brasil), Rosa Maria Rodrigues Pereira (Universidade de São Paulo, São Paulo, SP, Brasil), Samuel Katsuyuki Shinjo (Universidade de São Paulo, São Paulo, SP, Brasil)

\section{BACKGROUND}

Vessel imaging in Takayasu arteritis (TA) is often performed in clinical practice following clinical symptoms such as limb claudication. Conversely, the correlation between limb claudication and vessel imaging manifestations has not been assessed.

\section{MATERIALS AND METHODS}

This retrospective cohort, single-center study analyzed angiotomography, magnetic resonance imaging and/or Doppler vessel imaging in adult patients who fulfilled the 1990 ACR classification criteria for TA, between 1985 and 2018. All arterial narrowing or occlusions were registered. Simultaneously, all symptoms, especially limb claudication that prompted imaging investigation, were also collected, then cross-checked with imaging data to evaluate if limb claudication symptoms mean arterial lesions. P-value of less than 0.05 was considered statistically significant.

\section{RESULTS}

The cohort was comprised of 139 patients with TA, with a predominance of females (87.8\%) and white ethnicity (83.4\%). Mean age was 29.9 years and mean disease duration of 8.3 years when vessel imaging was performed. Limb claudication was reported in 42 (30.0\%) patients, of which were: $17.3 \%$ upper left limb (ULL), 12.2\% upper right limb (URL), 12.9\% lower left limb (LLL) and 12.2\% lower right limb (LRL). When cross-matched with imaging, the ULL and URL did not correlate to any vessel narrowing or occlusion imaging (i.e., ascending aorta, aortic arch and its branches: subclavial, carotid and/or vertebral). In contrast, the LRL claudication correlated to infra-renal aortic $(P=0.009)$, left iliac $(P=0.011)$ and right iliac $(P=0.001)$, whereas the LRL claudication correlated to infra-renal aortic $(P=0.001)$, left iliac $(P=0.033)$ and right iliac $(P<0.001)$ narrowing or occlusions. Moreover, the ULL and URL claudication symptoms correlated significantly with each other $(P<0.001)$.

\section{CONCLUSION}

Limb claudication is often an important symptom in patients with TA that triggers the radiographical search of arterial narrowing or occlusions. However, our data shows that upper limb claudication did not correlate with imaging findings; therefore, clinicians should rethink routine imaging evaluation based on this symptom. In contrast, lower limb claudication correlates to vessel imaging, especially with abdominal aortic and/or iliac narrowing or occlusions. 